"This is the peer reviewed version of the following article: Danny Haubold, Annett Reichhelm, Alexander Weiz, Lars Borchardt, Christoph Ziegler, Lydia Bahrig, Stefan Kaskel, Michael Ruck, Alexander Eychmüller (2016). The Formation and Morphology of Nanoparticle Supracrystals. Advanced Functional MaterialsVolume 26, Issue 27, July 19, 2016, Pages 4890-4895, which has been published in final form at DOI:10.1002/adfm.201600186.

This article may be used for non-commercial purposes in accordance with Wiley Terms and Conditions for Self-Archiving."

\title{
The Formation and Morphology of Nanoparticle Supracrystals
}

Danny Haubold, Annett Reichhelm, Alexander Weiz, Lars Borchardt, Christoph Ziegler, Lydia Bahrig, Stefan Kaskel, Michael Ruck, Alexander Eychmüller*

Danny Haubold, Annett Reichhelm, Alexander Weiz, Dr. Lars Borchardt, Dr. Christoph Ziegler, Dr. Lydia Bahrig, Prof. Dr. Stefan Kaskel, Prof. Dr. Michael Ruck, Prof. Dr. Alexander Eychmüller

Department of Chemistry and Food Chemistry, Technische Universität Dresden, 01062 Dresden, Germany

E-mail: Alexander.Eychmueller@chemie.tu-dresden.de

Keywords: substrate-affected growth, supracrystals, supercrystals, formation mechanism, silver nanoparticles, self-assembly

Supracrystals are highly symmetrical ordered superstructures built up from nanoparticles via self-assembly. While the NP assembly has been intensively investigated, the formation mechanism is still not understood. To shed some light onto the formation mechanism, we are using one of the most common supracrystal morphologies, the trigonal structures, as a model system to investigate the formation process in solution. To explain the formation of the trigonal structures and determining the size of the supracrystal seeds formed in solution, we introduce the concept of substrate-affected growth. Furthermore, we show the influence of the NP concentration on the seed size and extend our investigations from Ag towards Au.

\section{Introduction}

Nowadays, nanoparticles (NPs) can be easily synthesized in high quality via wet chemical approaches. Nevertheless, single particles cannot be used for most applications, such as solar cells, thermoelectric systems or field-effect transistors. Therefore, an assembly of particles is needed, while maintaining their special properties. Supracrystals are three dimensional 
superstructures built up via self-assembly of NPs, which are then called primary building blocks. ${ }^{[1,2]}$ In comparison to other superstructures, they exhibit regular, well-defined facets and shapes, while preserving the particular properties of the primary building blocks. ${ }^{[3,4]}$

The transformation mechanism from separated building units in solution to fully developed supracrystals on the substrate is one of the remaining open questions on the way to fully understand and control the supracrystal formation. Although, many investigations led to sophisticated preparation techniques ${ }^{[5-7]}$ and nanoparticle ordering ${ }^{[1,2,7,8]}$, the formation process in solution has not been sufficiently understood. The directed preparation of supracrystals for technical purposes demands the knowledge of the formation mechanism for the control of the resulting superstructures and of their properties.

Much effort has been put into the characterization of the final superstructures and the investigation of the NP assembly by many groups. We know from these studies that the particles can arrange into fcc, bcc or hcp superlattices showing a long range ordering of the particles. ${ }^{[8]}$ These supracrystals show a high symmetry and well-defined facets yielding octahedra $^{[7]}$, hexagonal plates ${ }^{[6]}$, five-armed stars ${ }^{[9]}$ and more complex twinned structures ${ }^{[10]}$. Furthermore, there are a lot of investigations dealing with the self-assembly of nanoparticles and the corresponding mechanism. Different models have been developed ranging from a hard sphere model ${ }^{[11,12]}$, where the NPs are assumed to be spherical objects of similar size, to soft sphere models, where the kind of ligand determines the self-assembly ${ }^{[13,14]}$. Also the driving force and the assembly probability on steps, holes or edges have been calculated. ${ }^{[15]}$ In this study, we achieved insight into the formation process in solution by investigating the morphology of the resulting supracrystals. Due to the large variety of reported morphologies, we decided to examine one of the most frequently observed symmetrical crystal shapes, which will be called trigonal supracrystal (see Figure 1) in the following. Additionally, we surveyed the influences of different preparation parameters on the resulting superstructures.

Trigonal Ag supracrystals have been prepared via gas-phase destabilization techniques (details can be found in the supporting information). On the basis of HRSEM, SAXS and TEM measurements, the concept of substrate-affected growth is introduced to explain the formation of trigonal shaped supracrystals yielding the size of the supracrystals, which are formed in solution. We will show that the self-assembly can be influenced by the preparation parameters such as concentration, temperature, NP size and size distribution leading to a 
control of the size of the supracrystals formed in solution. In order to evaluate the concept of substrate-affected growth, the investigations have been extended towards Au.

From the literature, we can conclude that trigonal supracrystals occurred, if there is on the one hand a fcc arrangement of the NPs and on the other hand a plane (substrate) surface.

Furthermore, octahedral supracrystals have been observed next to the trigonal structures. ${ }^{[16,17]}$ According to this information, we used a gas-phase destabilization method ${ }^{[7,10]}$, which reliable grants access to highly-symmetrical supracrystals with well-defined facets, to directly prepare trigonal shaped supracrystals. To ensure a slow destabilization of the NP solution, a nonsolvent slowly diffuses via the gas-phase into a non-stirred NP solution. A detailed description of the preparation procedure can be found in the experimental section.

\section{Results}

From the SEM images, we observe that mostly trigonal supracrystals have been built up (Figure $1 \mathrm{a}-\mathrm{c}$ ). These structures have already been presented in numerous publications. ${ }^{[5,7,18-21]}$ By SAXS, HRSEM, and TEM measurements, we can confirm that these supracrystals are built up from an assembly of the NPs into fcc superlattices with lattice parameters of about $10.4 \mathrm{~nm}$. These supracrystals show a high symmetry, well-defined facets and edges (see Figure 1). Details can be found in the supporting information.

The trigonal supracrystals show the characteristic facets of octahedra. The latter are a common form of fcc lattices independent of the size of the primary building blocks. ${ }^{[22]}$ The similarity of the trigonal supracrystals to octahedra can be seen in Figure $1 \mathrm{f}$. There, the red line represents the substrate surface. At first, an octahedron is placed on one (111) facet on the substrate. While increasing the size, which represents a growth of the supracrystals, but not moving the mass center, it becomes clear that the trigonal structures result. Three of the vertices of the deposited octahedron are lying on the substrate surface and transform into an edge during further growth. Furthermore, HRSEM images reveal the hexagonal dense assembly of the nanoparticles on the top of the trigonal structures, which represents one of the (111) facets of the initial octahedron. On the basis of these observations, we introduce the concept of substrate-affected growth, which provides an insight into the trigonal structure formation and contributes to a deeper understanding of the general supracrystals formation mechanism in solution. 
The supracrystal formation starts with the slow destabilization of the NP solution. At this stage, the NPs assemble in order to minimize the exposed surface to the solvent/precipitant leading to a seed formation. Therefore, the NPs mostly form a supracrystal seed, which grows, but still stays in solution. At a certain size, the supracrystal seed settles slowly on the substrate surface. The typical size of the as deposited supracrystals will be called the supracrystal seed size. Due to the fcc assembly of the nanoparticles, the shape of the seed is in most cases an octahedron. ${ }^{[22]}$ When settling to the ground, the octahedron will lay on one (111) facet. The assembly of further NPs on this particular (111) facet is not possible, leading to a substrateaffected growth. The resulting structures are trigonal supracrystals as shown in Figure $1 \mathrm{~d}$ )-f) from different viewing directions.

Complete octahedra are only obtained, when they are not lying on the substrate surface. An example is shown in Figure $1 \mathrm{c}$ ). There, an octahedron is placed between three other supracrystals. The main difference between these supracrystals is the orientation. While the trigonal structures have a (111) plane parallel to the ground, the vertex of the octahedral supracrystal points towards the substrate. This also provides evidence for the assumption that the trigonal structures originate from octahedral seeds.

The trigonal structures are a special kind of supracrystals and result from substrate-affected growth. Nevertheless, the seed formation in solution is the first step in the supracrystal formation and determines the final morphology, the size and properties of the resulting superstructure. Knowing and influencing the seed size is a crucial step to directly design and tune the properties of the resulting NP assembly. In this way, the trigonal structures can be used as a tool to determine the supracrystal seed size, which is the maximum size of the supracrystals formed in solution before settling to the ground.

In general, the substrate-affected growth can be divided into two steps (a scheme of the mechanism is shown in Figure 2). At the first phase, the seed is formed via self-assembly leading to an octahedral supracrystal, which grows on the substrate to a trigonal structure. Therefore, it is clear that the morphology and the size of the trigonal structures are connected to the size of the octahedron, which settled to the ground. Herein, the substrate-affected growth concept allows calculating the supracrystal seed size. The used model is shown in Figure 3. The small octahedron represents the supracrystal seed, which lays on one (111) facet on the substrate. The mass center of the octahedron has a distance $h$ to the ground. As the 
crystal growths, the substrate-affected growth mechanism is operative, which leads to trigonal superstructures. The yellow part represents the trigonal supracrystal that is built, while the red part represents the missing units to complete a virtual octahedron. Every plane is a (111) facet having the same growth speed in every non-restricted direction. A virtual octahedron, which would be formed by a non-substrate-affected growth, can be constructed by combining the yellow and the red part. The mass center of the supracrystal seed and the virtual octahedron stays the same, which can be used to determine the seed size $c_{S}$ with equation 1 (detailed derivation in the supporting information).

$$
c_{s}=\frac{d-0.5 d^{*}}{0.5 d^{*}} c_{T}
$$

The edge length $c_{S}$ is a characteristic value for the supracrystal seed and can be calculated by measuring the edge length of the trigonal structure $c_{T}$, the distance between the upper and lower edge $d$ and the distance $d^{*}$ between the upper edge and the tip of the virtual octahedron (example shown in S 5). To get an easier understanding of the size of the seed, the volume of the octahedron can be calculated with equation 2 .

$$
V_{S}=\frac{\sqrt{2}}{3} c_{s}^{3}
$$

In order to control the seed formation, the preparation parameters such as temperature, concentration and NP size and size distribution have been varied leading to tunable seed sizes and a control of the resulting superstructures.

An increase of the NP concentration leads to bigger trigonal structures (Table $1 \&$ Figure 4), ranging from $\mathrm{c}_{\mathrm{T}}=4.0 \mu \mathrm{m}$ up to $16.0 \mu \mathrm{m}$. Furthermore, the seed size is increasing from $2.4 \mu \mathrm{m}$ to $6.7 \mu \mathrm{m}$. A further increase of the NP concentration leads only to thin films, while lowering leads to small aggregates without any symmetry. The values for the seed size have been confirmed by SEM. There are only octahedra, which are smaller than the determined seed size on the substrate surface (example shown in S 6). We assume that a too high concentration leads to a fast precipitation of the NPs. Furthermore, there are too many particles, which coat the ground and also cover the supracrystal seeds. On the other hand, if the concentration is too low, only a few unsymmetrical aggregates can be found. This implies that there is no seed formation in solution as the mean distance between the particles is too large.

In-situ DLS measurements show evidence of the calculated seed sizes. A detailed description can be found in the supporting information. Briefly, an ethanol phase has been added on top of a NP solution leading to a slow destabilization over time forming supracrystals. ${ }^{[23]}$ The 
change of the hydrodynamic diameter over time is shown in S 7. At the beginning only the hydrodynamic diameter of the single NPs can be measured. After $10 \mathrm{~h}$, a second peak at around $700 \mathrm{~nm}$ occurs indicating the formation of small aggregates. Within a few minutes, this peak shifts towards $2 \mu \mathrm{m}$ and is stable at this position for several hours until the complete destabilization and precipitation of the NPs. Larger aggregates have not been observed. These values correspond very well to the calculated critical seed sizes shown here. Similar results have already been shown for PbS supracrystals in a former publication. ${ }^{[24]}$

Changing the destabilization temperature $\left(0{ }^{\circ} \mathrm{C}, \mathrm{RT}, 40{ }^{\circ} \mathrm{C}\right)$ affects only the formation time (5-6 weeks, 3-4 weeks, 1 week), but has no effect on the supracrystal size or the seed size. It should be noted that increasing the temperature to above $40{ }^{\circ} \mathrm{C}$ or lowering to below $0{ }^{\circ} \mathrm{C}$ while using the gas-phase destabilization method, leads to bigger aggregates without any symmetry. Supracrystals could only be obtained within this small temperature range.

Differently sized nanoparticles $(5.5,8.8,11.3$ and $12.5 \mathrm{~nm})$ have been used to investigate the influence of the size of the nanoparticles on the supracrystal formation. Particles which are smaller than $6 \mathrm{~nm}$, form supracrystals with well-defined facets, while bigger particles mostly lead to more complex structures and layers of nanoparticles. In order to shed more light on these observations, a solution of differently sized nanoparticles has been used. Thus, $5.5 \mathrm{~nm}$ and $12.5 \mathrm{~nm}$ NPs have been mixed. Supracrystals, on the top of a thin film of NPs, have been obtained (S 8). The investigation of the thin film and the supracrystals showed that there was a size-selective separation during the destabilization process. Supracrystals are built only from the small particles, while the $12.5 \mathrm{~nm}$ sized NPs can be found in the thin film coating the substrate.

Considering two different NP sizes (5.5 and $12.5 \mathrm{~nm}$ ), the mass and colloidal stability are different. During the slow destabilization of the NP solution, the bigger particles precipitate first, followed by the smaller ones (Figure 5). From the experiments with nearly monodisperse $12.5 \mathrm{~nm}$ sized NPs, we can conclude that they mostly arrange in thin films. In the first phase, the bigger particles coat the ground followed by a destabilization of the smaller NPs. As the smaller NPs aggregate in the solution, which is known from the experiments with nearly monodisperse NP solutions, they form supracrystal seeds. These seeds settle to the ground and grow as described above. In this way the NPs are separated. 
Furthermore, the concept of substrate-affected growth has been extended to Au supracrystals. Thus, $5 \mathrm{~nm}$ sized Au NPs have been synthesized. The surfactant is oleylamine, which also has been used for the Ag NPs. The Au supracrystals show the same morphologies as the Ag pendants. Besides a few octahedra or twinned structures mostly trigonal structures are observed. For these, the seed size $c_{S}$ and the volume $V_{S}$ have been determined (Table 2). The $\mathrm{Au}$ NPs show the same trigonal superstructures as gained from Ag. Also the critical seed size is comparable.

\section{Conclusion}

Here, trigonal Ag supracrystals have been prepared by the gas-phase-destabilization method and used as a model system to get a deeper insight into the formation mechanism in solution. These structures are built up from a fcc superstructure as confirmed by SAXS, HRSEM and TEM measurements. On the basis of these investigations, we introduced the concept of substrate-affected growth, which is able to explain the morphology of the trigonal supracrystals. From this model, we can conclude that supracrystal seeds are formed in solution and grow until they reach a certain size and settle to the ground followed by a further growth on the substrate. The supracrystals seed size can be calculated by our proposed model. By changing the NP concentration the seed size can be tuned and the resulting structures are influenced.

\section{Experimental Section}

Materials: Acetone (p.A.) and ethanol were purchased from VWR, borane-tert-butylamine complex (TBAB) from ABCR, gold(III) chloride trihydrate (99.99\%), methanol (99\%), octadecene (ODE, 90\%), oleic acid (90\%), oleylamine (90\%), iso-propanol (99\%), n-propanol (99\%), silver nitrate (99.99\%), 1,2,3,4-tetrahydronaphthalene (tetraline, 99\%) and toluene (p.A.) from Sigma-Aldrich.

Ag NPs: Ag NPs have been synthesized according to a modified preparation route of Chen et al. ${ }^{[25]} 150 \mathrm{mg}$ AgNO3 are dissolved in $20 \mathrm{ml}$ ODE and $6 \mathrm{ml}$ oleylamine. The mixture was purged with Ar and heated to $160{ }^{\circ} \mathrm{C}$. After $2 \mathrm{~h}$ it was cooled to $150{ }^{\circ} \mathrm{C}$ and stirred for another

$6 \mathrm{~h}$. The reaction was quenched by a fast cool down to room temperature. The small particles 
were removed by a size-selective precipitation with defined amounts of acetone, washed and redispersed in toluene.

Au NPs: Au NPs have been prepared by a synthesis of Jana and Peng. ${ }^{[26]} 200 \mathrm{mg}$ of HAuCl4 3 $\mathrm{H} 2 \mathrm{O}$ has been dissolved in $11 \mathrm{ml}$ of tetraline and $11 \mathrm{ml}$ olelyamine and purged with nitrogen for $15 \mathrm{~min}$. In a glovebox $87 \mathrm{mg}$ tert-butylamine borane complex (TBAB) has been dissolved in $1 \mathrm{ml}$ tetraline and $1 \mathrm{ml}$ oleylamine. This mixture has then been quickly injected at room temperature. The mixture was stirred for another $60 \mathrm{~min}$. The NPs have been precipitated by acetone, washed twice and dispersed in toluene.

Gas-phase destabilization method: Briefly, in a $2 \mathrm{ml}$ vial a silicon wafer has been placed as a substrate. $1 \mathrm{ml}$ of the corresponding nanoparticle solution was added. This vial has then been placed into a $5 \mathrm{ml}$ vial, which was filled with $2 \mathrm{ml}$ of the precipitant. Toluene and octane have been used as solvents and ethanol, methanol, i-propanol, n-propanol and acetone as precipitants. This vial was sealed and stored at a certain temperature. Depending on the solvent/non-solvent pair, the destabilization process takes up to four weeks.

Instruments: High-resolution scanning electron microscopy (HRSEM) has been performed with a SU8020 (Hitachi) using the semi inlense detector $(\mathrm{Ua}=30 \mathrm{kV})$. Standard scanningelectron microscopy (SEM) has been made by a DSM 982 GEMINI (Zeiss) using an acceleration voltage between 3 and $10 \mathrm{kV}$. Small angle X-ray scattering (SAXS) experiments were performed on a Bruker Nanostar $(\mathrm{Cu} \mathrm{K \alpha} ; \lambda=1.5406 \AA)$ with a position sensitive HiStar detector. The samples were prepared in flat plate geometry and the sample to detector distance was $105 \mathrm{~cm}$, to cover a range of $2 \Theta$ up to $3^{\circ}$. Transmission electron microscopy (TEM) has been performed using a FEI Tecnai F30 at $300 \mathrm{kV}$ and a field emitter source. Therefore, the samples have been prepared via drop-casting onto a Lacey-Grid (graphene oxide).

\section{Supporting Information}

Supporting Information is available from the Wiley Online Library or from the author. 


\section{Acknowledgements}

This work was financially supported by the Deutsche Forschungsgemeinschaft through the research training group NANO- AND BIOTECHNOLOGIES FOR PACKAGING OF ELECTRONIC SYSTEMS DFG 1401/2 and the financial support from the European Research Council (ERC-2013-AdG AEROCAT).

Received: ((will be filled in by the editorial staff))

Revised: ((will be filled in by the editorial staff)) Published online: ((will be filled in by the editorial staff))

[1] M. Niederberger, H. Cölfen, Phys. Chem. Chem. Phys. 2006, 8, 3271.

[2] R. Q. Song, H. Cölfen, Adv. Mater. 2010, 22, 1301.

[3] L. Zhou, P. O’Brien, J. Phys. Chem. Lett. 2012, 3, 620.

[4] L. Bahrig, S. G. Hickey, A. Eychmüller, CrystEngComm 2014, 16, 9408.

[5] N. Goubet, H. Portalès, C. Yan, I. Arfaoui, P.-A. Albouy, A. Mermet, M.-P. Pileni, J. Am. Chem. Soc. 2012, 134, 3714.

[6] C. B. Murray, C. R. Kagan, M. G. Bawendi, Science (80-. ). 1995, 270, 1335.

[7] P. Simon, E. Rosseeva, I. Baburin, L. Liebscher, S. G. Hickey, R. Cardoso-Gil, A. Eychmüller, R. Kniep, W. Carrillo-Cabrera, Angew. Chemie Int. Ed. 2012, 51, 10776.

[8] A. Courty, J. Richardi, P.-A. Albouy, M.-P. Pileni, Chem. Mater. 2011, 23, 4186.

[9] S. M. Rupich, E. V Shevchenko, M. I. Bodnarchuk, B. Lee, D. V Talapin, J. Am. Chem. Soc. 2010, 132, 289.

[10] L. Bahrig, D. Haubold, F. Röder, S. G. Hickey, A. Eychmüller, MRS Proc. 2014, 1705, mrss 14.

[11] B. de Nijs, S. Dussi, F. Smallenburg, J. D. Meeldijk, D. J. Groenendijk, L. Filion, A. Imhof, A. van Blaaderen, M. Dijkstra, Nat. Mater. 2014, 14, 56.

[12] B. Gates, J. Light. Technol. 1999, 17, 1956.

[13] D. V Talapin, E. V Shevchenko, M. I. Bodnarchuk, X. Ye, J. Chen, C. B. Murray, Nature 2009, 461, 964.

[14] P. Simon, L. Bahrig, I. a Baburin, P. Formanek, F. Röder, J. Sickmann, S. G. Hickey, A. Eychmüller, H. Lichte, R. Kniep, E. Rosseeva, Adv. Mater. 2014, 26, 3042.

[15] W. Kossel, Ann. Phys. 1934, 413, 457.

[16] H. Portalés, N. Goubet, S. Sirotkin, E. Duval, A. Mermet, P. A. Albouy, M. P. Pileni, Nano Lett. 2012, 12, 5292. 
[17] N. Goubet, J. Richardi, P. a. Albouy, M. P. Pileni, J. Phys. Chem. Lett. 2011, 2, 417.

[18] N. Goubet, J. Richardi, P.-A. Albouy, M.-P. Pileni, J. Phys. Chem. B 2013, 117, 4510.

[19] C. Yan, I. Arfaoui, N. Goubet, M.-P. Pileni, Adv. Funct. Mater. 2013, 23, 2315.

[20] Y. Yang, W. Wang, T. Chen, Z.-R. Chen, ACS Appl. Mater. Interfaces 2014.

[21] S. Wang, S. Sato, K. Kimura, Chem. Mater. 2003, 15, 2445.

[22] A. Mersmann, Dry. Technol. 1995, 13, 1037.

[23] D. V. Talapin, E. V. Shevchenko, A. Kornowski, N. Gaponik, M. Haase, A. L. Rogach, H. Weller, Adv. Mater. 2001, 13, 1868.

[24] L. Bahrig, Dissertation, TU Dresden (Dresden, Germany), 05, 2014.

[25] M. Chen, Y.-G. Feng, X. Wang, T.-C. Li, J.-Y. Zhang, D.-J. Qian, Langmuir 2007, 23, 5296.

[26] N. R. Jana, X. Peng, J. Am. Chem. Soc. 2003, 125, 14280. 


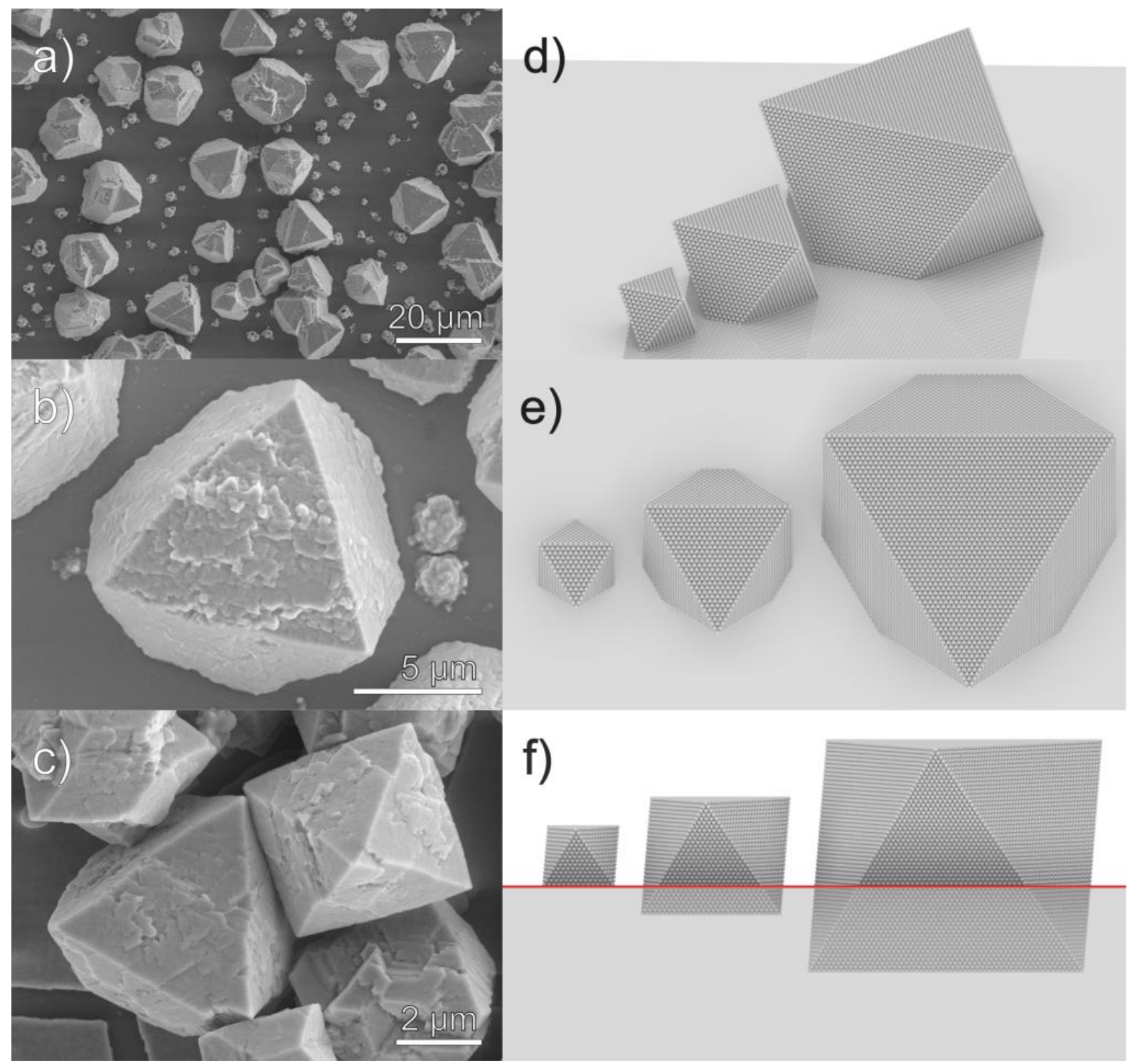

Figure 1: a)-c) Supracrystals prepared by the gas-phase destabilization method. The most common supracrystal morphology is the trigonal structure. This can be explained by using the concept of substrate-affected growth. The models d)-f) show the process of a growing octahedral seed on the substrate surface leading to the shown trigonal structures. The red line in f) represents the substrate surface. The material, which is above the red line, is formed during the further assembly. The material under the red line, in the grey part, represents the material, which would be built up if there would be no restriction. 
Phase 1: Destabilization

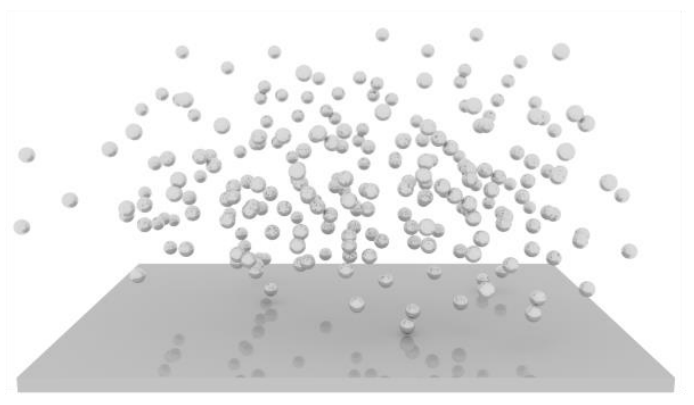

Phase 4: Restricted growth

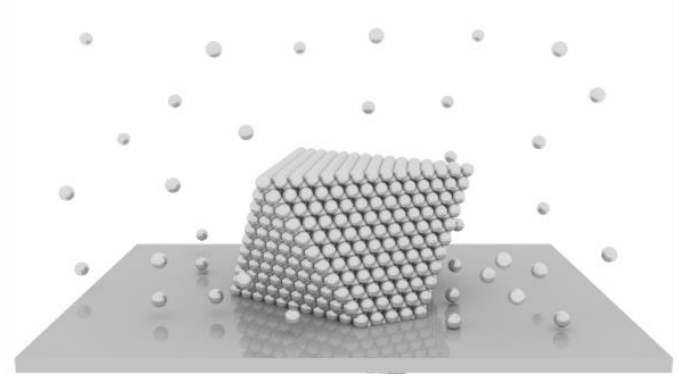

Phase 2: NP assembly in solution
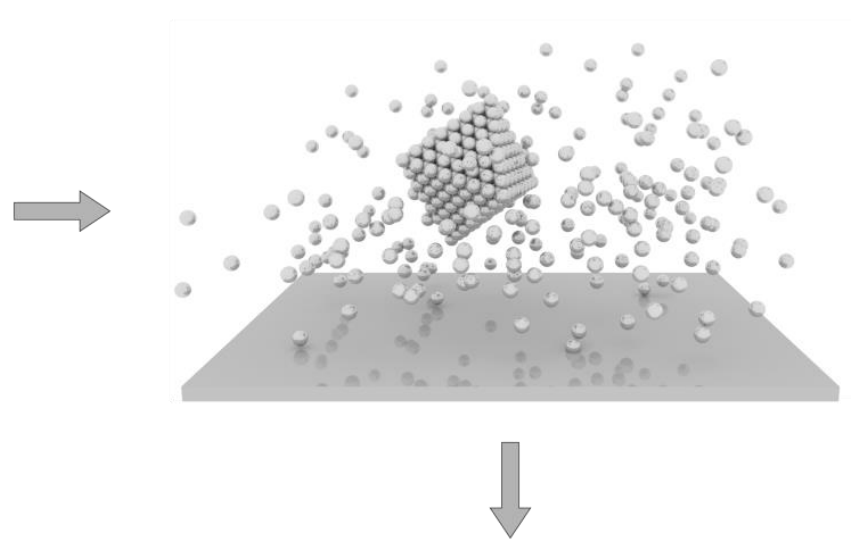

Phase 3: Seed precipitation

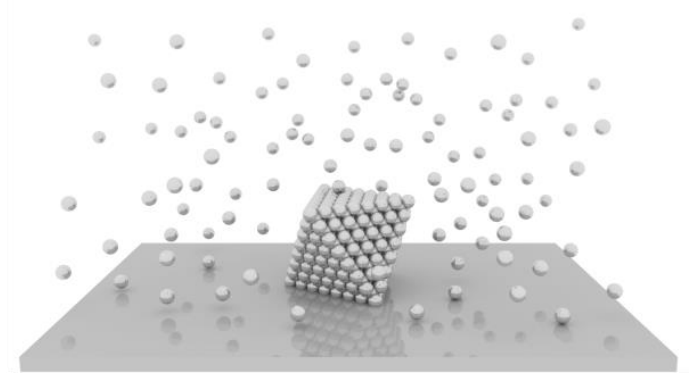

Figure 2: Substrate-affected growth mechanism. In the first phase, the NPs are destabilized. In the second step, the particles assemble to minimize the exposed surface to the surrounding media. In this step the first supracrystal seeds are built. Due to the $f c c$ arrangement, mostly octahedra are formed. The seeds are growing and getting heavier. At a certain seed size, they settle down to the substrate surface and lay on one (111) facet. In the last phase, the remaining NPs assemble onto the seed, which leads to a growth of the seed in all directions at the same speed, while the plane, which points towards the substrate, is not available for any further attachment. Due to this substrate-affected growth, supracrystals with trigonal shapes result. 

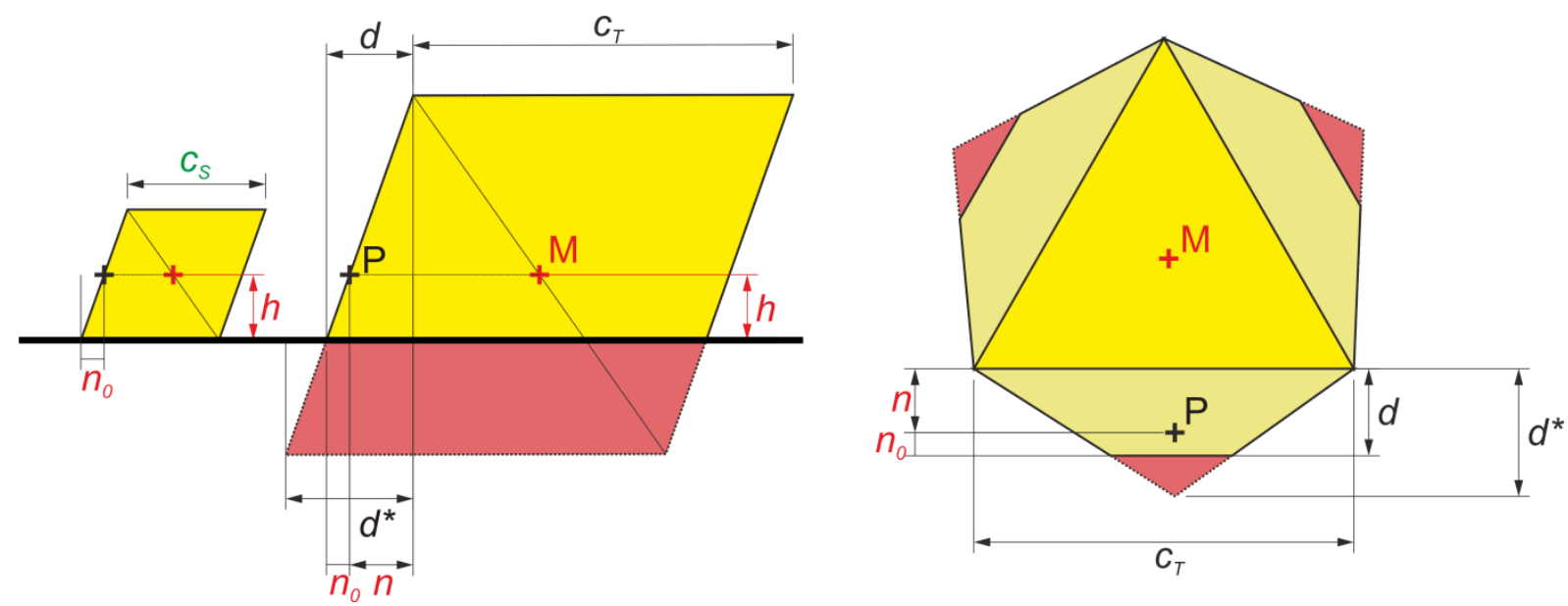

Figure 3: Geometrical model to calculate the supracrystal seed size. The black line represents the substrate surface. The seed (small yellow octahedron) lays on one (111) facet and is growing due to the further destabilization of the NPs. The resulting trigonal structure is placed next to the seed. The red parts are missing from a virtual octahedron, which needs to be constructed to calculate the seed size $c_{S}$. Values in black can be measured directly or with the help of the construction of a virtual octahedron (red parts) from the trigonal structures. Red values are required to determine $c_{S}$. 

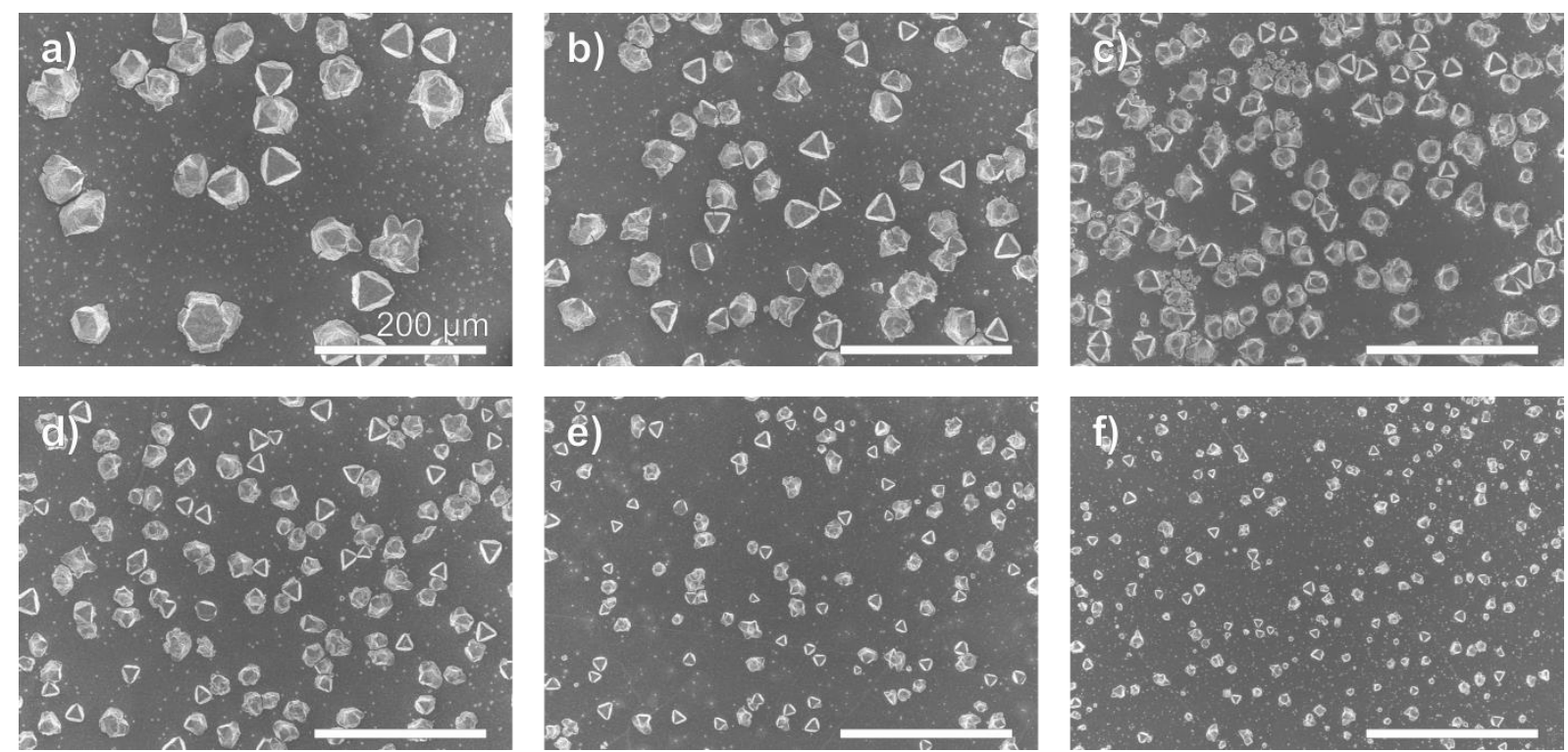

Figure 4: Influence of the NP concentration on the supracrystal size. While decreasing the NP concentration from a) to f), the supracrystals decrease in size. Furthermore, also the seed size is decreasing. The scale bars are $200 \mu \mathrm{m}$ in all images. The concentrations correspond to the values shown in Table 1. 

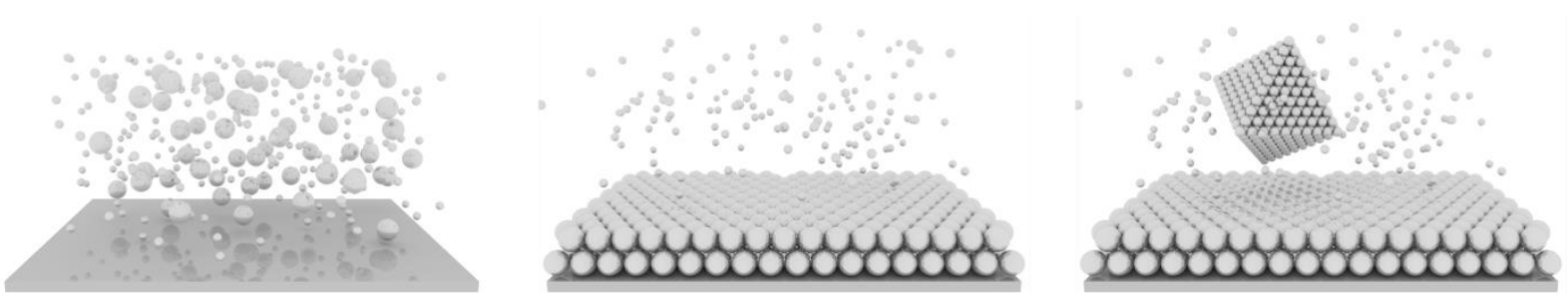

Figure 5: Starting from a solution which contains two differently sized NPs, the big NPs are destabilized first. Therefore, they coat the ground, while the smaller ones remain in the solution and form supracrystal seeds, which then settle to the ground and underlie the substrate-affected growth. 
Table 1: Concentration-depending values for $c_{T}, c_{S}$ and $V_{S}$ for $\mathrm{Ag}$ supracrystals. By increasing the NP concentration, the size of the supracrystals and of the seeds is increasing.

\begin{tabular}{llll}
\hline $\begin{array}{l}\text { NP conc. } \\
\left(10^{15} \mathrm{NP} / \mathrm{ml}\right)\end{array}$ & $\begin{array}{l}c_{T} \\
(\mu \mathrm{m})\end{array}$ & $\begin{array}{l}c_{S} \\
(\mu \mathrm{m})\end{array}$ & $\begin{array}{l}V_{S} \\
\left(\mu \mathrm{m}^{3}\right)\end{array}$ \\
\hline 5.65 & 16.0 & 6.7 & 142 \\
4.52 & 11.0 & 4.7 & 49 \\
3.39 & 11.6 & 4.9 & 55 \\
2.83 & 8.0 & 3.2 & 15 \\
2.26 & 5.7 & 2.4 & 6.5 \\
1.13 & 4.0 & 2.6 & 8.3
\end{tabular}

Table 2: Average values for $c_{T}, c_{S}$ and $V_{S}$ of $\mathrm{Ag}$ and Au supracrystals. The radius of the used NPs is shown for comparison and includes the size of the inorganic core and the ligand shell. The NP concentration $\left(1.13 \cdot 10^{15} \mathrm{NP} / \mathrm{ml}\right)$ is the same in the two samples.

\begin{tabular}{lllll}
\hline material & $\begin{array}{l}r_{N P} \\
(\mathrm{~nm})\end{array}$ & $c_{T}(\mu \mathrm{m})$ & $c_{S}(\mu \mathrm{m})$ & $V_{S}\left(\mu \mathrm{m}^{3}\right)$ \\
\hline $\mathrm{Ag}$ & 3.8 & 4.0 & 2.6 & 8.3 \\
$\mathrm{Au}$ & 3.5 & 4.5 & 2.5 & 7.4
\end{tabular}




\section{The table of contents entry}

Trigonal supracrystals are an ideal system to investigate the self-assembly of nanoparticles in solution into highly-symmetrical structures. The proposed substrate-affected growth mechanism allows determining the size of the crystal seeds built up in solution. The seed size is tuned by the change of the preparation parameters.

Keyword: substrate-affected growth, supracrystals, supercrystals, formation mechanism, silver nanoparticles, self-assembly

Danny Haubold, Annett Reichhelm, Alexander Weiz, Lars Borchardt, Christoph Ziegler, Lydia Bahrig, Stefan Kaskel, Michael Ruck, Alexander Eychmüller*

The Formation and Morphology of Nanoparticle Supracrystals

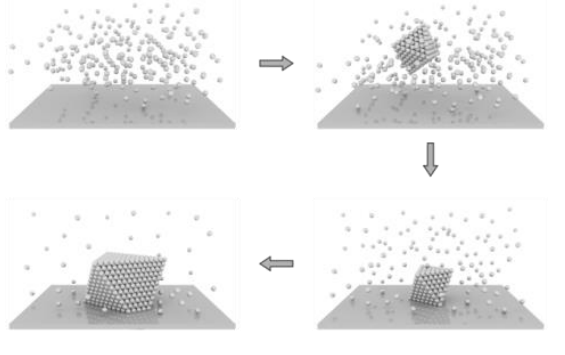




\section{Supporting Information}

\section{The Formation and Morphology of Nanoparticle Supracrystals}

Danny Haubold, Annett Reichhelm, Alexander Weiz, Lars Borchardt, Christoph Ziegler, Lydia Bahrig, Stefan Kaskel, Michael Ruck, Alexander Eychmüller*

\section{Characterization of the NP assembly}

In order to investigate the arrangement of the nanoparticulate building blocks within the supracrystal, SAXS measurements $\left(\mathrm{Cu} \mathrm{K}_{\alpha} ; 1.5406 \AA\right)$ have been performed (S1). Three reflections were obtained at $2 \Theta=1.48^{\circ}$ (strongest signal), $1.7^{\circ}$ (shoulder), and $2.81^{\circ}$ (weak signal). Assuming the supracrystal to be assembled of spherical particles of uniform size, either face-centered cubic ( $f c c$, corresponding to $\mathrm{Cu}$-type structure), hexagonal close packing ( $h c p$, corresponding to Mg-type structure), or a body-centered cubic ( $b c c, \mathrm{~W}$-type structure) packing is possible. Using the free software Scatter, theoretical patterns of $f c c, h c p$ and $b c c$ were calculated and fitted to the experimental data, assuming that the strongest reflection, matches the signal of highest intensity of the experimental data (S 1). The refinement of the super cell results the lattice vector $a$ with $10.4 \mathrm{~nm}(f c c), 7.8 \mathrm{~nm}(h c p)$ and $4.5 \mathrm{~nm}$ (bcc). Only a $f c c$ packing can describe the experimental data properly. According to the $f c c$ superstructure, the lattice parameter $a$ is $10.4 \mathrm{~nm}$.

To confirm the results obtained by SAXS, single NP layers have been prepared via dropcasting on a TEM grid (S4). The TEM images show the hexagonal ordering of the NPs in a single layer as seen from the $\{111\}$ planes of octahedral supracrystals. The distance between the centers of the particles has been determined as $7.5 \mathrm{~nm}$, which corresponds to the size of the inorganic core and two times the ligand length. Assuming an (111)-plane in a $f c c$ superlattice, the cell parameters can be calculated by equation 4 , where $k$ is the distance between two NPs within one layer. The lattice parameter $a$ has been determined as $10.6 \mathrm{~nm}$, which corresponds to the value, obtained by SAXS.

$$
\sqrt{2} \cdot k=a
$$

We can conclude that the mesocrystals are built up from Ag NPs, which are arranged in a $f c c$ lattice. The lattice parameter $a$ of the mesocrystals has been determined as $10.4 \mathrm{~nm}$ by SAXS 
measurements and confirmed by a simulation of the inner structure. HRSEM revealed the $(111)_{S L}$ and (100) SL arrangement of the NPs at the planes and tip of an octahedral mesocrystal, respectively. On the basis of these observations, we introduce the concept of substrateaffected growth in the next paragraph.

S 1: Indexing and calculation of theoretical reflections assuming $f c c, h c p$ and $b c c$ superlattices. The theoretical reflections have been calculated by the free software SCATTER. The marked values represent the signals with the highest intensities and have been used to fit the experimental results to a superlattice cell.

\begin{tabular}{|c|c|c|c|c|c|}
\hline \multicolumn{2}{|c|}{$\begin{array}{c}\text { fcc } \\
a=10.4 \mathrm{~nm}\end{array}$} & \multicolumn{2}{|c|}{$\begin{array}{c}\boldsymbol{h} \boldsymbol{c p} \\
a=7.8 \mathrm{~nm}\end{array}$} & \multicolumn{2}{|c|}{$\begin{array}{c}\text { bcc } \\
a=4.5 \mathrm{~nm}\end{array}$} \\
\hline $2 \theta$ & reflection & $2 \theta$ & reflection & $2 \theta$ & reflection \\
\hline 1.48 & 111 & 1.31 & 100 & 1.48 & 110 \\
\hline 1.7 & 200 & 1.38 & 002 & 2.07 & 200 \\
\hline 2.4 & 220 & 1.48 & 101 & 2.55 & 211 \\
\hline \multirow[t]{4}{*}{2.81} & 311 & 2.26 & 110 & 2.94 & 220 \\
\hline & & 2.46 & 103 & & \\
\hline & & 2.65 & 112 & & \\
\hline & & 2.69 & 201 & & \\
\hline
\end{tabular}

a)

b)
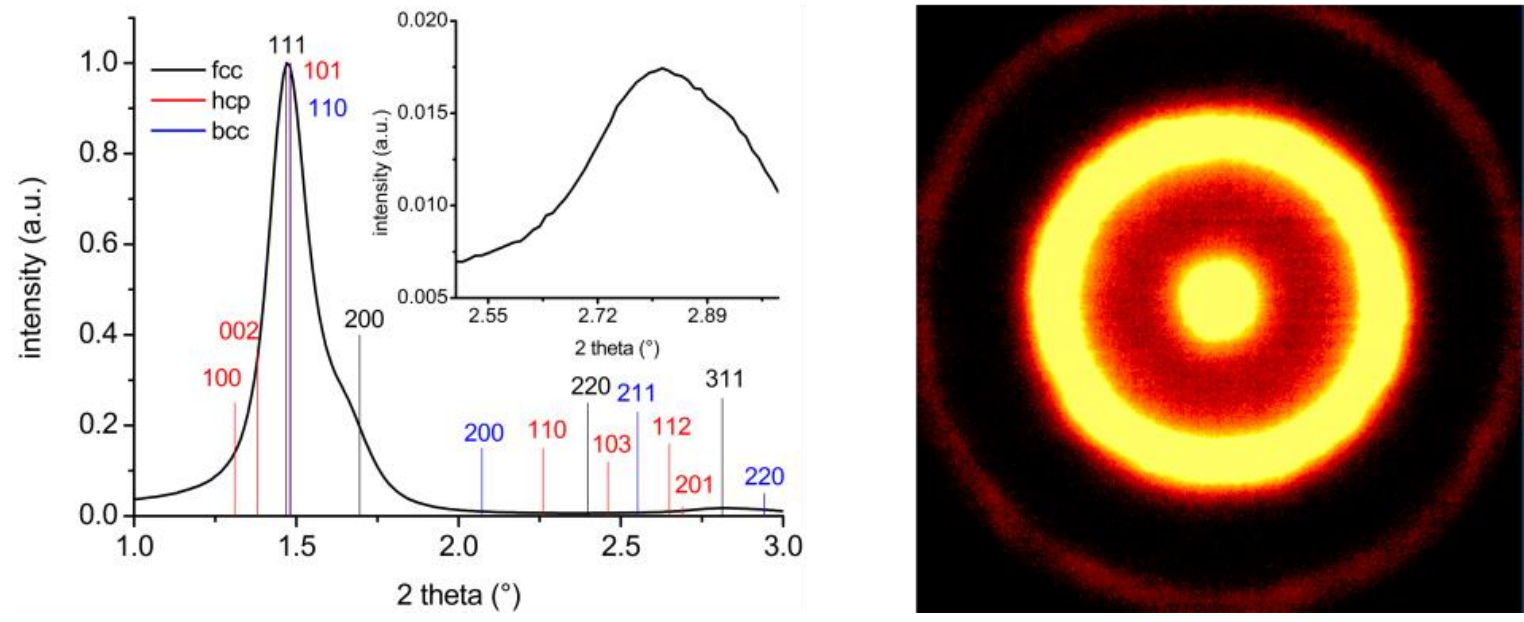

S 2: SAXS measurements $\left(\mathrm{Cu} \mathrm{K}_{\alpha} ; 1.5406 \AA\right)$ from Ag supracrystals. The diffractogram a) shows the integrated intensities from the scattering image $b$ ). The inset reveals the reflection at higher angles with less intensity. The lines indicate the theoretical reflections from $f c c$ (black), $h c p$ (red) and $b c c$ (blue) superlattices. Only the $f c c$ signals coincide with the experimental observations, indicating the kind of superstructure. 


\section{HRSEM investigations}

A single octahedron has been characterized in regard to its faces by HRSEM, which is shown in $S 3$ a)-c). The $\{111\}$ planes of the octahedron show a hexagonal arrangement of the NPs as expected from $f c c$ assemblies. Furthermore, the tip of the octahedron reveals a face of tetragonal ordered NPs, which corresponds to the $\{100\}$ plane. Generally, the preferred direction of growth is in $\langle 100\rangle$ resulting in that almost exclusively the $\{111\}$ planes can be observed. The model shown in $\mathrm{S} 3 \mathrm{~d}$ ) was created by a $f c c$ arrangement of spheres in an octahedral shape and reveals the same assembly.
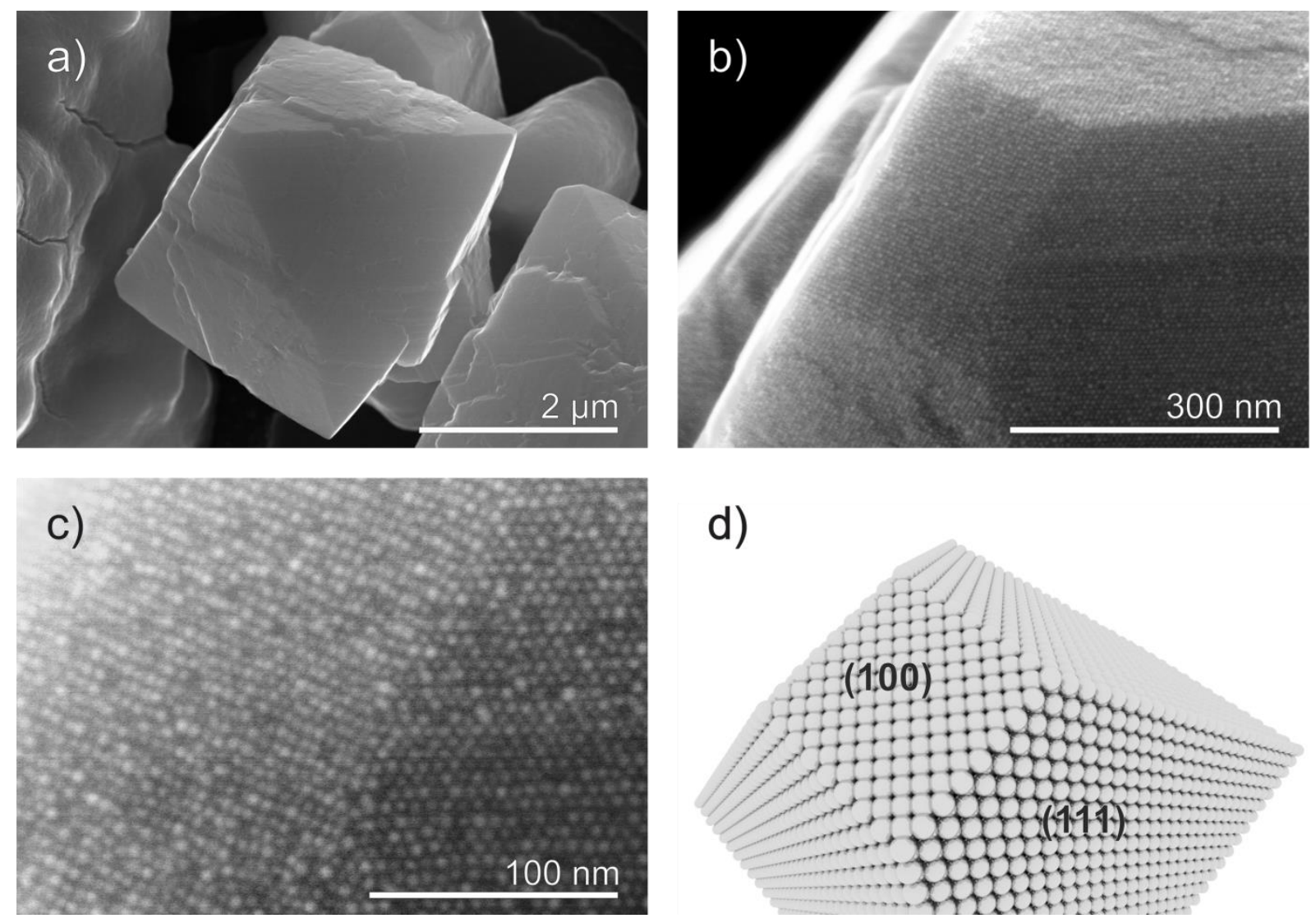

S 3: HRSEM images from a single octahedron a) with view on $\{111\}$ and $\{100\}$ facets. At higher magnifications b) and c), the arrangement of the NPs is seen on the side planes and the tip. The side planes show the hexagonal arrangement, while the tip is truncated and reveals the cubic assembly of the NPs. These images prove the $f c c$ arrangement of the NPs within the supracrystal. The model structure in d) shows the theoretical assembly of a $f c c$ lattice. The tip of the model reveals the cubic arrangement of the NPs, while the side planes represent the $\{111\}$ assembly. 


\section{TEM investigations}

Single NP layers have been prepared via drop-casting on a TEM grid (S 2). The TEM images show the hexagonal ordering of the NPs in a single layer as seen from the $\{111\}$ planes of octahedral supracrystals. The distance between the centers of the particles has been determined as $7.5 \mathrm{~nm}$, which corresponds to the size of the inorganic core and two times the ligand length. Assuming an (111)-plane in a $f c c$ superlattice, the cell parameters can be calculated by equation 4 , where $k$ is the distance between two NPs within one layer. The lattice parameter $a$ has been determined as $10.6 \mathrm{~nm}$, which corresponds to the value, obtained by SAXS.

$$
\sqrt{2} \cdot k=a
$$

We can conclude that the supracrystals are built up from Ag NPs, which are arranged in a $f c c$ lattice. The lattice parameter $a$ of the supracrystals has been determined as $10.4 \mathrm{~nm}$ by SAXS measurements and is confirmed by a simulation of the inner structure. HRSEM revealed the (111)SL and (100)SL arrangement of the NPs at the planes and at the tip of an octahedral supracrystal, respectively. On the basis of these observations, we introduce the concept of substrate-affected growth in the next paragraph to shed some light onto the supracrystal formation mechanism. 

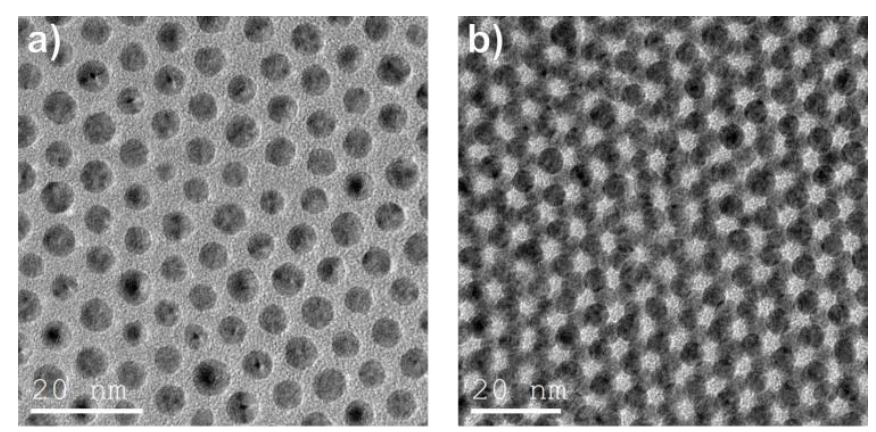

S 4: NPs have been self-assembled on a TEM grid to simulate the structure of the mesocrystal. The single NP layers a) reveals the hexagonal NP arrangement. Assuming the hexagonal arrangement to be the (111) plane, the lattice parameter $a$ has been determined as $10.6 \mathrm{~nm}$. Furthermore, the stacking of the hexagonal layers has been observed $b$ ). 


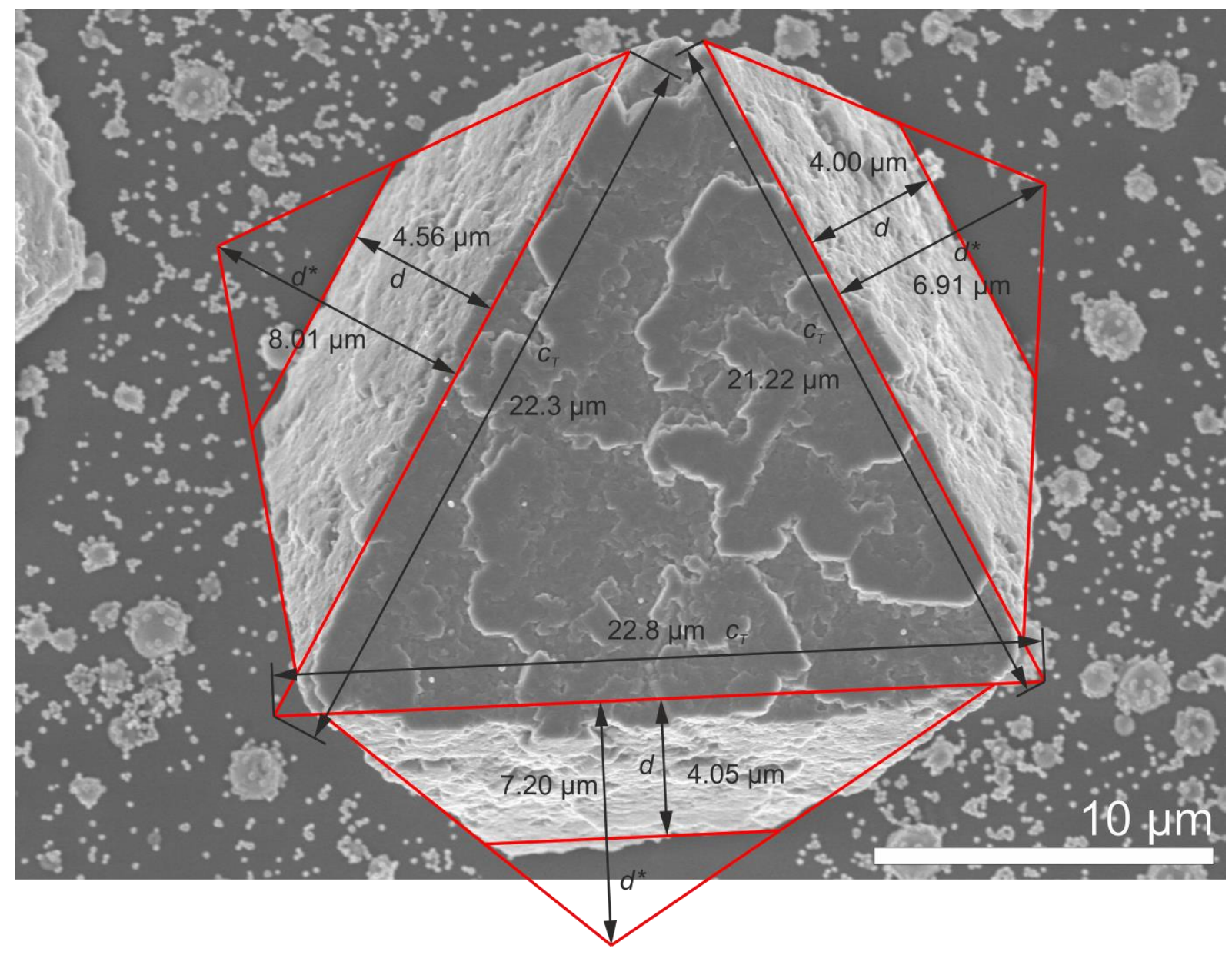

S 5: Example for the measurement of the seed size by determining $c_{T}, d$ and $d^{*}$ for every edge of the triangular structure. 


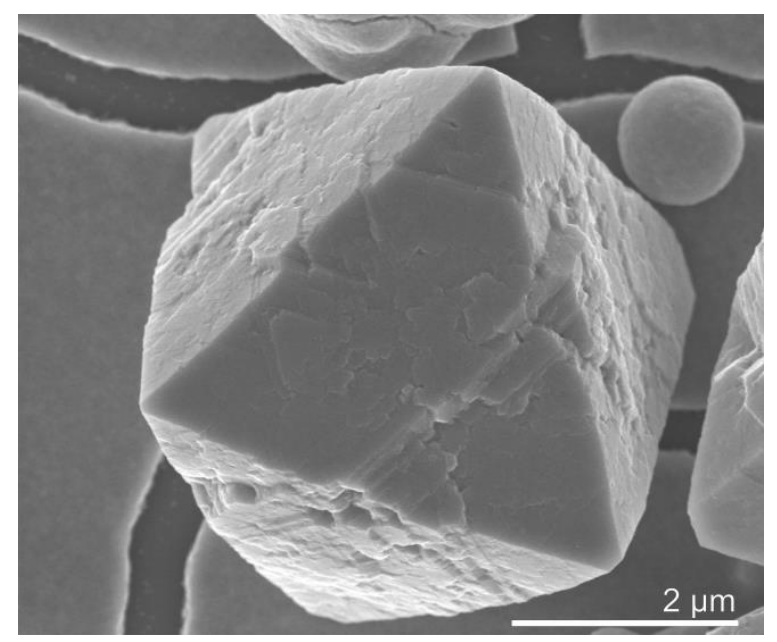

S 6: Octahedral mesocrystal, which is smaller than the determined seed size lying on one (111) facet. It is assumed that this crystal was built in solution. The starting substrate-affected growth is indicated at the right tip of the superstructure, which starts to become truncated, due to the further growth of the mesocrystal. 


\section{In-situ DLS investigations}

It is important to note that the gas-phase-destabilization method is a continuous changing system. Over time a non-solvent diffuses via the gas-phase into the NP solution (details in the experimental section). Thus, the polarity of the liquid phase is changing over time leading to the aggregation of the NPs. In order to investigate the different supracrystal formation stages, this process has been simulated by adding an ethanol phase on top of the NP solution. This leads to a slow diffusion of the non-solvent into the NP solution and thus to a slow destabilization over time. ${ }^{[23]}$ Considering a DLS measurement, the refractive index of the solvent and non-solvent should be close. Considering all the mentioned points, toluene (1.497)/ethanol(1.361) have been chosen as solvent/non-solvent.

The results are shown in S 7. At the beginning, only the diameter of the Ag NPs can be measured, which is $7 \mathrm{~nm}$. After $10 \mathrm{~h}$, a second signal occurs at a hydrodynamic diameter of about $700 \mathrm{~nm}$. Within a few minutes, this peak shift towards $2 \mu \mathrm{m}$ indicating the formation of the supracrystals. Within the following $15 \mathrm{~h}$, there is no shift of the peak. After $24 \mathrm{~h}$, the hydrodynamic diameter dramatically decreases within a few minutes indicating the complete destabilization (all NPs have been precipitated).

At the beginning ( $0 \mathrm{~min}$ to $602 \mathrm{~min}$ ), there is a slow diffusion of the ethanol to the toluene phase leading to a slow destabilization. Thus, during the first $10 \mathrm{~h}$ no changes occur. At the second stage (692 min-1553 min), first assemblies are formed, which increase in size over a small time frame. When the aggregates reach the critical seed size, they precipitate and cannot be measured by the DLS system (from $1560 \mathrm{~min}$ ). While supracrystals of more than few micrometers do not stay in solution, the further growth must take place at the substrate leading to the characteristic trigonal supracrystals. This observation proves the substrateaffected growth mechanism.

A detailed discussion of the DLS experiments and the formation mechanism of the supracrystals for PbS NPs can be found in a former publication. ${ }^{[24]}$ 


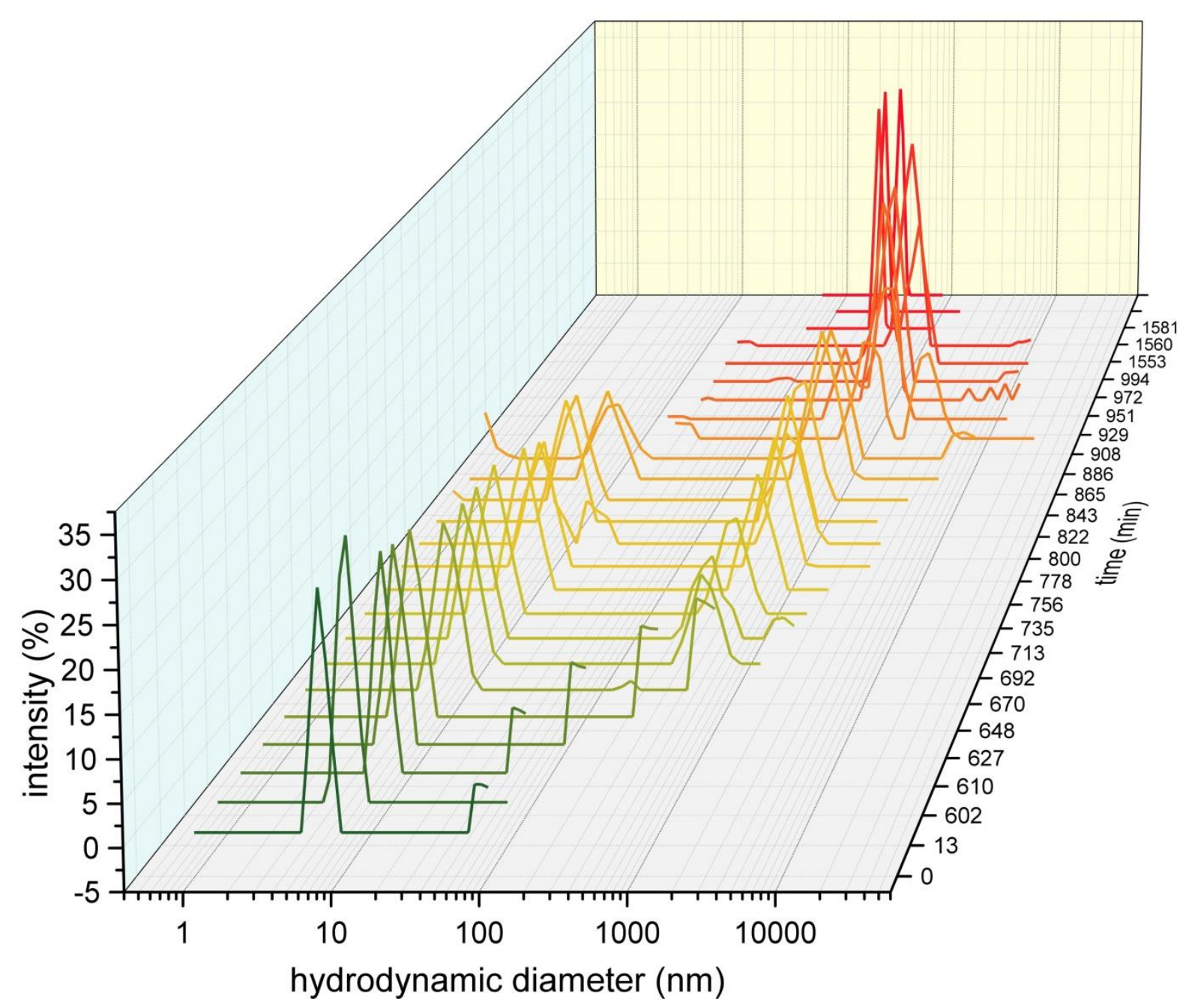

S 7: In-situ DLS measurements of the destabilization of a Ag NP solution. At the beginning, only the size of the NPs can be detected. After $10 \mathrm{~h}$, first assemblies are formed, which increase in size over a few minutes. After $24 \mathrm{~h}$, the destabilization is complete and all NPs have been precipitated. No aggregates larger than $2 \mu \mathrm{m}$ can be observed showing proof to the concept to substrate-affected growth. 


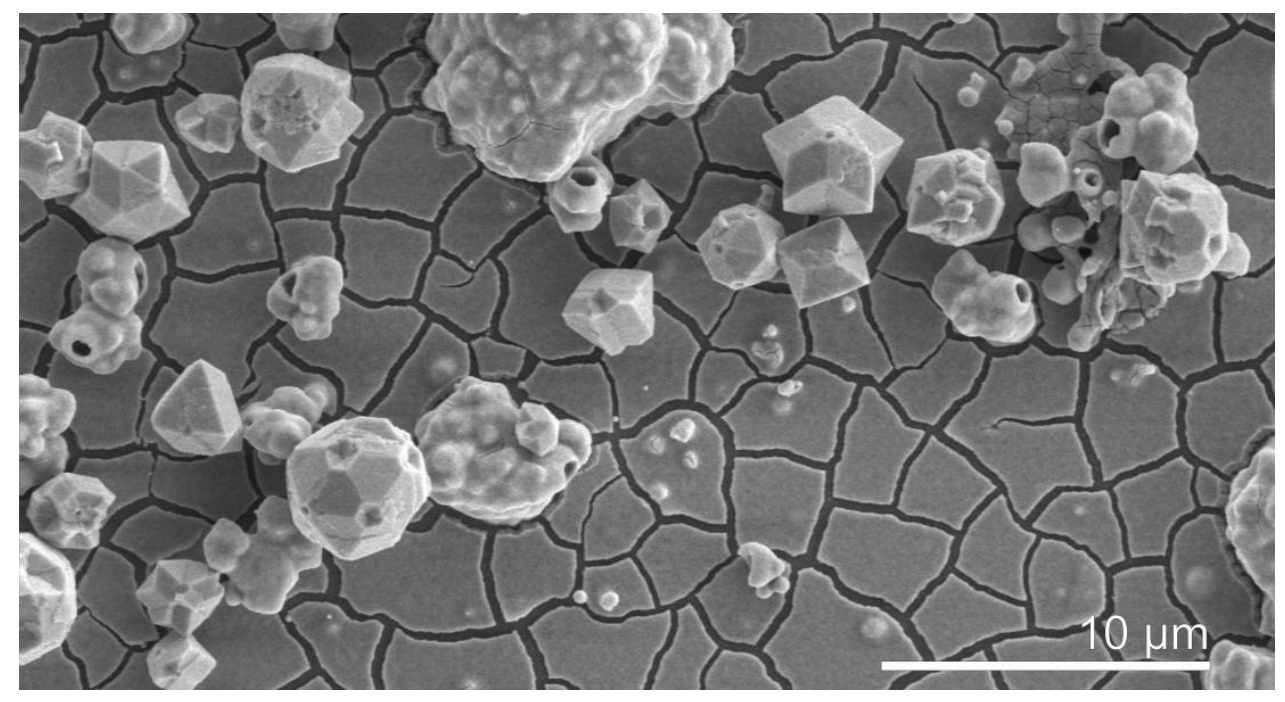

S 8: Mesocrystals prepared by mixing two different sized NPs. The substrate surface is coated with the bigger NPs, while the mesocrystals are formed by the smaller ones. Even in this complex system, the substrate-affected growth applies. 


\section{Derivation of $c_{S}$}

At the beginning of the substrate-affected growth process, the superstructure seed is placed onto the substrate while its center of mass $\mathrm{M}$ is located in a distance $h$ above the substrate. The projection of $\mathrm{M}$ onto a side face leads to the projection point $\mathrm{P}$ in the middle of the height of the face in the distance $n_{0}$ from the vertex contacting the substrate (see Figure 3).

When the seed grows, the center of mass of the virtual octahedron remains in the same position. Hence, the values of $\mathrm{h}$ and $n_{0}$ stay the same and conserve the information of the original size of the seed. The half height $n$ of the large virtual octahedron increases proportionally with the edge length $c_{T}$ and is required for the calculation of the seed size $c_{S}$ :

$$
\frac{c_{S}}{c_{T}}=\frac{n_{0}}{n}
$$

$c_{T}$ can be measured in the images of the triangular structures. As the mass center lies directly at the half height of the virtual octahedron, $n$ equals the half of $d^{*}$, which can be measured by the construction of the virtual octahedron.

$$
n=0.5 d^{*}
$$

As the mass center of the virtual octahedron stays at the same height, $\mathrm{n}_{0}$ can be calculated by:

$$
\begin{gathered}
n_{0}=d-n \\
n_{0}=d-0.5 d^{*}
\end{gathered}
$$

$d$ is the distance from the upper edge to the bottom edge, representing the cutting of the virtual octahedron, due to the substrate-affected growth. Due to the used model system it is required that $d-0.5 d^{*}$ is positive. Some crystals have been observed, which showed a negative value. This can be explained by the complexity of the construction of the virtual octahedron. By putting equation 8 and 6 into 5 the superstructure seed size can be calculated as:

$$
c_{S}=\frac{d-0.5 d^{*}}{0.5 d^{*}} c_{T}
$$


